\section{P141 Designing a Nutrition Intervention to Profile Metabolomic, Microbiome and Vascular Health in Young Adults: FRUVEDomic Study}

Melissa Olfert, DrPH, RD, melissa.olfert@mail.wvu.edu, West Virginia University, PO Box 6108, Ag. Sc. Building G016, Morgantown, WV 26506-6108; O. Famodu, MS, RD; D. Kesner, BS; J. White, BS; M. Barr, BS; C. Warner; M. Dehlin, BS; M. Buerkle, BS; M. Downs, PhD, MLS; C. Moore, BS; P. Murray, MD; J. McFadden, PhD; C. Cuff, PhD; S. Colby, PhD, RD, University of Tennessee; J. Stabile Morrell, PhD, University of New Hampshire; M. Olfert, PhD, West Virginia University

Objective: To design a multi-disciplinary nutrition education approach based on the 2010 Dietary Guidelines for Americans, using nutrition education, culinary toolkit and one-on-one counseling, to impact metabolomics, cardiovascular, and microbiome health in "at risk" young adults (18-28 years) for metabolic syndrome (metS).

Design, Setting and Participants: Researchers from disciplines (nutrition, metabolomics, microbiome, cardiovascular, microcirculation and physiology) designed a nutrition education approach targeting young adults "at risk" for metS to participate in an 8-week nutritional interventional study at West Virginia University. Subjects $(n=37)$ were randomized into one of three nutritional intervention groups; "Fruved" (50\% fruit \& vegetable); "Fruved+LowCHO" (low refined carbohydrate); and "Fruved+LowFat" (low fat).

Outcome Measures and Analysis: Anthropometrics, surveys, venous blood samples, stool samples, arterial stiffness and body composition were collected. A two-hour group nutrition education including basic nutrition for the prescribed intervention, culinary tool kit, budget and grocery shopping tips were delivered at baseline followed by weekly individual consultations with $\mathrm{RD}$, using food logs, food pictures and receipt management, to assess adherence and cost of the intervention.

Results: Participants (male $=16$; female $=21$ ) "at risk" of metS , 37\% were considered high risk $(n=14)$, followed by $35 \%$ medium risk $(n=13), 22 \%$ medium/high risk $(n=8)$ and $5 \%$ low risk $(n=2)$. Of the participants, $54 \%$ $(n=20)$ were from the Appalachian region and $64 \%$ $(n=24)$ were Caucasian. Linear trends among all groups for fruit and vegetable consumption are observed, averages ranged from 0-1 cups of fruit at baseline to 2-4 cups at midway of the intervention. Vegetable consumptions has shown similar trends, going from 1-2 cups to 2-3 cups of vegetables. Additionally, lower percentage of calories from fat are observed among the low-fat intervention arm from baseline (36\% at baseline to $26 \%$ at week 4 ) and lower empty calories (1065-baseline, 313-week 4) and higher amounts of fiber (17g-baseline, 24g-week 4) have been seen for the low-refined carbohydrate group.

Conclusions and Implications: Designing multi-discipline approaches to delivering the nutritional guidelines is the next translational step to educating how a healthy diet plays a higher impact on health (i.e. higher fruit and vegetable consumption, low-fat, low-refined carbohydrate).

Funding: NIH.

\section{P142 Efficacy of Teen Battle Chef Program to Shift the Academic Performance and Health Behaviors in NYC High School Students}

Lynn Fredericks, BA, lynn@familycookproductions.com, FamilyCook Productions, 330 East 43rd Street, Suite 704, New York, NY 10017; H. Park, MS, Columbia University, Institute of Human Nutrition; N. Sliva, MS; E. Irvin, MPH, HealthCorps; S. Hayes, PhD; Judith Wylie-Rosett, RD, EdD, Albert Einstein College or Medicine

Objective: Teen Battle Chef (TBC) uses culinary and nutrition education to promote behavioral change by empowering youth on multiple levels. This study examines the impact of the program on NYC high school students' food behaviors, leadership, attendance and academic performance.

Design, Setting and Participants: We examined the food behaviors, leadership traits, attendance and academic performance of TBC students in 14 NYC HealthCorps high schools. 176 students participated in the intervention and 40 students in the comparison group. Pre and post surveys were conducted with students from both i groups. Additionally, school performance data in a subset of students from the previous school year in the same schools were examined to determine whether participation affected school performance.

Outcome Measures and Analysis: Academic data (2012-13) from a total of 88 TBC's from 2012-13 were compared to the entire school population. These included attendance, SAT scores, and graduation.

Results: The TBC group significantly increased their overall dietary quality $(0.114 ; \mathrm{p}=0.03)$ compared with the control group $(0.006 ; p=0.90)$. An indication of leadership development, participants had significantly greater improvement in discussing the value of local foods with others $(\mathrm{t}=-3.31 ; \mathrm{p}<0.01)$. Compared with previous year's cohort, the mean SAT scores for TBC participants were significantly higher than overall school scores. Additionally, TBC participants attendance rate was 95\%, compared to the school attendance rate of $86 \%$.

Conclusions and Implications: This study provides strong evidence that TBC helps participants develop leadership, teamwork, culinary skills, nutrition knowledge and self-efficacy. TBC students also improve their academic performance and attendance and are motivated to succeed in school as a result of their participation.

Funding: HealthCorps.

\section{P143 Does a Didactic Curriculum In Nutrition Influence the Sodium Intake by Students?}

Lalitha Samuel,PhD, Lalitha.Samuel@lehman.cuny.edu, Lehman College, City University of New York, 250 Bedford Park Boulevard W, Bronx, NY 10468; N. DeLaCruz, BS

Objective: To measure the 24-hour urinary sodium content of students enrolled in the Nutrition major, during 